\title{
P043: Re-emergence of influenza a $\mathrm{H} 1 \mathrm{~N} 1$ in Saudi Arabia
}

\author{
NA Alzahrani ${ }^{*}$, S Al Johani ${ }^{2}$ \\ From 2nd International Conference on Prevention and Infection Control (ICPIC 2013) \\ Geneva, Switzerland. 25-28 June 2013
}

\section{Introduction}

Influenza A H1N1 is a novel influenza virus that is of swine source. Clinical manifestations of this virus vary from mild respiratory symptoms to fatal respiratory or/ and cardiovascular complications. The new influenza A H1N1 pandemic was first identified in April 2009 in the United States and Mexico, and then spread globally. In September 10 2010, the World Health Organization announced that the influenza A H1N1 pandemic had moved into the post-pandemic period and is no longer considered a dangerous global disease. On the $15^{\text {th }}$ of August 2010, the Ministry of Health in Saudi Arabia declared that the cases of influenza A H1N1 in 2010 have drastically declined to 874 cases with no deaths or serious complications.

\section{Objectives}

In this study, we will shed light on the number of cases of influenza A H1N1 infections in Saudi Arabia from the months of September to November 2012, and describe the cases where influenza A H1N1 was the cause of death in these patients, and the underlying comorbidities that may have led to severe complications or death.

\section{Methods}

This is a retrospective study in which we reviewed our laboratory records for cases that were tested for influenza A H1N1 in the months of September, October, and November 2012. All samples were nasopharyngeal aspirates and were tested using The Xpert Flu ${ }^{\circledR}$ assay and performed on Cepheid ${ }^{\circledR}$ GeneXpert medical device system.

\section{Results}

67 patient samples were tested for influenza A H1N1 over the period from September to November 2012. Overall, there were 19 positive samples, 13 of those samples were positive for influenza A H1N1, 6 were positive for influenza $\mathrm{A}$, and none tested positive for influenza $\mathrm{B}$. Two patients were suffering from other co-morbidities and expired as a result of influenza A H1N1 infection.

\section{Conclusion}

Vaccination for influenza A H1N1 is widely available in Saudi Arabia. However, some people are resisting vaccination. This resistance may have caused the re-emergence of influenza A H1N1 in Saudi Arabia. Public awareness should be improved to aware people of the benefits of vaccination and the consequences of not getting vaccinated.

\section{Disclosure of interest \\ None declared.}

\section{Author details}

${ }^{1}$ College of Applied Medical Sciences, King Saud bin Abdulaziz University for Health Sciences, Saudi Arabia. ${ }^{2}$ Department of Pathology and Laboratory Medicine, King Abdulaziz Medical City, Riyadh, Saudi Arabia.

Published: 20 June 2013

doi:10.1186/2047-2994-2-S1-P43

Cite this article as: Alzahrani and Al Johani: P043: Re-emergence of influenza a H1N1 in Saudi Arabia. Antimicrobial Resistance and Infection Control 2013 2(Suppl 1):P43. 